\title{
Zum Konzept des Netzwerks im englischen Vertragsrecht ${ }^{1}$
}

\section{Einleitung}

Vor 20 Jahren beschäftigte das englische Vertragsrecht ein schwieriges dogmatisches Problem an der Schnittstelle zwischen Schuld- und Deliktsrecht. ${ }^{2}$ Das Problem war entstanden, weil das Prinzip der Relativität von Verträgen (einer der Eckpfeiler des klassischen Vertragsrechts) ${ }^{3}$ mit der Bereitschaft der Rechtsprechung zusammentraf, im Anschluss an die Entscheidung des House of Lords im Fall Junior Books Ltd v Veitchi Co $\mathrm{Ltd}^{4}$ eine Fahrlässigkeitshaftung für reine Vermögensschäden anzuerkennen. Die Vertragsrechtler haderten mit einer strikten Anwendung des Relativitätsprinzips, die nicht nur berechtigte Ansprüche Dritter ausschloss, sondern auch berechtigten Erwartungen in Geschäftsbeziehungen entgegenlief. Die Deliktsrechtler hingegen befürchteten, dass Junior Books die Schleusen öffnen und den fahrlässig Handelnden einer unüberschaubaren Haftung gegenüber einer Unzahl von Klägern aussetzen würde. Und die Verfechter von »Contorts « schließlich waren beunruhigt über die Art und Weise, in der Junior Books den Klägern zu erlauben schien, das Relativitätsprinzip zu umgehen und ihre Ansprüche nicht auf Delikt, sondern auf Vertrag zu stützen.

Die Schwierigkeiten lösten sich durch dogmatische Justierungen auf beiden Seiten des Problems. Einerseits kam in Murphy v Brentwood District Council ${ }^{5}$ ein besonders zusammengesetzter Spruchkörper des Haus of Lords aus sieben Richtern in einer neuen Auslegung von Junior Books zu einer viel restriktiveren Fahrlässigkeitshaftung für reine Vermögensschäden. Andererseits wurde nach anhaltenden Angriffen von Seiten der Rechtsprechung wie der Kommentatoren der Relativitätsgrundsatz durch einen Akt des Gesetzgebers, den Contracts Rights of Third Parties Act 1999, gelockert. Das englische Recht war somit nicht ins neue Jahrtausend aufgebrochen, ohne die Befürchtungen von Vertrags- wie Deliktsrechtlern zu besänftigen. Mit der neuen Kombination aus Lockerung und Verschärfung wurden die Klagen von Dritten in Richtung Vertrag anstatt Delikt gelenkt.

Noch auf dem Höhepunkt der Schwierigkeiten wurde als Lösung unter anderem vorgeschlagen, dass zusammenhängende Verträge (wie etwa im Baurecht die Konstruktionsverträge in Junior Books und Murphy, aber auch im Bereich des Transportrechts, wo ähnliche Probleme entstanden waren) als eine spezielle Gruppe behandelt werden

1 Übersetzung aus dem Englischen: Dan Wielsch. Ich bin den Teilnehmern der Konferenz »Contractual Networks: Legal Issues of Multilateral Cooperation« und meinen Kollegen am King's College dankbar, John Phillips für hilfreiche Kommentare und Gespräche während der verschiedenen Etappen an der Arbeit für diesen Aufsatz.

2 Vgl. Roger Brownsword (2000) Contract Law: Themes for the Twenty-First Century, London: Butterworths.

3 Dunlop Pneumatic Tyre Co Ltd v Selfridge \& Co Ltd [1915] AC 847.

4 [1983] 1 AC 520.

5 [1991] 1 AC 398. 
sollten, als »Netzwerke« mit speziellen Regeln. ${ }^{6}$ In diesem Zusammenhang wurde behauptet, dass die Idee von Netzwerken und entsprechenden Netzwerkverträgen eine gezielte Abschwächung des Relativitätsprinzips erleichtern könnte, um so den berechtigten Erwartungen der Vertragsparteien Rechnung zu tragen. ${ }^{7}$ Vom Vorliegen eines Netzwerks (und entsprechenden Netzwerk-Rechtsfolgen) sollte grundsätzlich dann auszugehen sein, wenn eine bestimmte Gruppe von Verträgen durch einen übergreifenden Zweck (wie etwa durch Bezug auf ein und dasselbe geschäftliche Projekt) verbunden würde. Typischerweise existiert dann ein Hauptvertrag (oder eine Mehrzahl von Hauptverträgen), mit dem die anderen Verträge direkt oder indirekt verbunden sind. ${ }^{8}$ Dieser Ansatz impliziert nicht nur, dass es den Parteien eines Hauptvertrages der eigentlich nach den Regeln für Netzwerke beurteilt werden müsste - unbenommen ist, die Netzwerk-Regeln abzubedingen, sondern auch, dass die Parteien in Zusammenhängen, in denen nicht von einem Netzwerk auszugehen ist, gleichwohl frei sind, ihren Vertrag den Netzwerk-Regeln zu unterstellen.

Selbstverständlich ist die beschriebene Idee eines Netzwerks nicht völlig neu. Im englischen privaten Baurecht sind in der laufenden Vertragsbeziehung spezielle Regeln anwendbar, wenn die Ausführung einem sogenannten »Bau-Schema « folgt; die Idee einer vertraglichen Verbindung wurde von einigen Gerichten benutzt, um Klagen aus Delikt dort zu verhindern, wo dies mit in Wahrheit bestehenden Netzwerk-Erwartungen inkonsistent gewesen wäre ${ }^{9}$ und schließlich ist der Netzwerk-Gedanke dem Konzept der »Vertragsgruppen« (groupes de contrats) nicht unähnlich, das in Frankreich von der Ersten Zivilkammer des Cour de Cassation entwickelt worden ist. ${ }^{10}$ Bedenkt man vor diesem Hintergrund, dass die Lockerung des Relativitätsprinzips durch die Gesetzgebung von 1999 sowohl Klägern wie Beklagten größere Freiheiten in vertraglichen Geschäftsbeziehungen verschafft hat, so stellt sich die Frage, ob es einen Grund gibt, die Idee von Netzwerk-Verträgen erneut zu betrachten. Sollten wir stattdessen das »Netzwerk« besser als eine Idee zu den Akten legen, deren Zeit niemals wirklich gekommen war und die heute definitiv vorbei ist? ${ }^{11}$

Gegen die Annahme, den Netzwerk-Gedanken der Geschichte zu überantworten, behaupte ich, dass er aus mindestens zwei Gründen hilfreich bleibt. Erstens wird das Vertragsrecht dort, wo es die vernünftigen Erwartungen der Parteien einer Transaktion

6 Vgl. John Adams und Roger Brownsword (1989) »From Jarvis to Junior Books: Tortuous and Tortious Constructions«, 5 Construction Law Journal, 3 ff. und dies. (1990) »The Aliakmon and the Hague Rules«, Journal of Business Law, 23-35.

7 John Adams und Roger Brownsword (1990) »Privity and the Concept of a Network Contract«, 10 Legal Studies, 12-37 und dies. (1995) Key Issues in Contract, London: Butterworths, Kapitel 5.

8 Vgl. John Adams und Roger Brownsword (1995) (Fn. 6) 149.

9 Vgl. etwa Marc Rich and Co AGv Bishop Rock Marine Co Ltd: The Nicholas H [1996] AC 211.

10 Vgl. Barry Nicholas (1992) The French Law of Contract, Oxford: Clarendon, 172-177.

11 Für einen kritischen (netzwerk-orientierten) Kommentar zu den Vorschlägen der Reformkommission (Law Com. Nr. 242, Juli 1996) vgl. John Adams, Deryck Beyleveld und Roger Brownsword (1997) »Privity of Contract - the Benefits and the Burdens of Law Reform«, 60 Modern Law Review, 238-264. 
zu schützen und die Berechtigung solcher Erwartungen mit Rücksicht auf die bestehende Geschäftspraxis zu beurteilen hat, auf die Idee eines Netzwerks zurückzugreifen haben, um die operativen Erwartungen der Vertragsparteien zu formulieren - mit entsprechenden Folgen für die vom Gericht oder Schiedsgericht zu treffende Entscheidung. In diesem Zuge könnte dem Netzwerk-Konzept eine noch weitergehende Anwendung eröffnet sein: Durch die Berücksichtigung von Verbrauchern an den Endpunkten von integrierten Vertriebssystemen; bei der Lösung von wettbewerbsrechtlichen Fällen, die die Vertragsrechtler beschäftigen; ${ }^{12}$ bei der Lösung konzernrechtlicher Fälle, in denen erhebliche Differenzen bestehen zwischen der Vereinbarung eines Kreditmodells und seiner tatsächlichen Durchführung; ${ }^{13}$ und möglicherweise durch die Anwendung auf die Praxis dezentralen Kontrahierens, wie sie auf elektronischen Marktplätzen begegnet. Sofern wir, zweitens, ein Netzwerk als ein von den Vertragsparteien selbst gewähltes Rechtsregime betrachten, besitzen wir den Schlüssel, um zwischen spezifisch vertraglichen und delikts-ähnlichen Mechanismen zur Regulierung von Transaktionen zu unterscheiden. Wie sich herausstellen wird, kann die Bindung an Netzwerk-Regeln in Fällen, in denen sich die Vertragsparteien bewusst für ein Netzwerk-Regime entschieden haben, nicht als obskurer Sonderfall abgetan werden, sondern gerät geradezu zum idealtypischen Beispiel einer vertraglichen Verpflichtung.

Ich gehe in drei Schritten vor. Als erstes beschreibe ich ein dogmatisches Problem, das durch die Lockerung des Relativitätsprinzips im Zusammenhang mit der richterlichen Kompetenz zur Inhaltskontrolle von Haftungsausschluss- und Haftungsbegrenzungsklauseln (oder, allgemeiner, unfairen Vertragsklauseln) verursacht wird. Kurz gesagt, entsteht das Problem, wenn ein Gericht entscheiden muss, ob eine Klausel, die in einem Vertrag zwischen A und B enthalten ist, angemessen ist, obwohl der Konflikt eigentlich zwischen A und $\mathrm{C}$ besteht. Ein solches Problem wird häufig zwischen Unternehmern auftreten; es kann aber auch entstehen, wenn Verbraucher involviert sind. In beiden Fällen wurzelt die Schwierigkeit darin, dass der richtige Referenzrahmen für die Frage nach der Angemessenheit schwierig zu finden ist, solange Verträge als isolierte bipolare Transaktionen anstatt als innerhalb eines Netzwerks verbundene Verträge aufgefasst werden. Ähnliche Problemen entstehen, wenn ein begünstigter Dritter eine Vertragsklausel anders auffasst als die Parteien des Hauptvertrags. Verallgemeinernd lässt sich sagen, dass Fälle, in denen das Vertragsrecht einen auf das Geschäft zwischen A und B bezogenen Kontext voraussetzt, immer dann schwierig werden, wenn C (mit einer durchaus anderen Transaktionshistorie) ins Spiel kommt. Auch wenn die Dogmatik teilweise revidiert worden sein mag und begünstigte Dritte inzwischen nicht völlig unberücksichtigt bleiben, so stellt das Auftauchen Dritter die herkömmliche Dogmatik immer wieder vor unerwartete Probleme. Jedenfalls in einigen solcher Fälle könnte in der Idee des Netzwerks die Lösung liegen.

12 Vgl. etwa Clarke v Earl of Dunraven and Mount-Earl, the Satanita [1897] AC 59.

13 Vgl. etwa Amalgamated Investment and Property Co Ltd (in liquidation) v Texas Commerce International Bank Ltd [1982] QB 84. 
Zweitens unterscheide ich zwischen zwei unterschiedlichen Rechtfertigungsgründen, um Vertragsparteien an Regelsets für Transaktionen zu binden, sei es, dass diese Regeln die Wirkungen des Vertrages auf Dritte oder die Gültigkeit von Haftungsbegrenzungen betreffen. Eine Rechtfertigungsmöglichkeit bezieht sich auf den Konsens der Parteien in dem Sinne, dass diese aus freien Stücken ein bestimmtes Set von Regeln gewählt haben (wie Spieler die übereinkommen, die Regeln eines bestimmten Spiels zu beachten; oder wie »Netizens «, die sich einem bestimmten Verhaltensregime unterwerfen, sobald sie einen bestimmten Sektor des Cyberspace betreten). ${ }^{14}$ Das ist der Idealtypus einer vertraglichen Verpflichtung. Die andere Rechtfertigungsmöglichkeit gründet nicht auf dem Parteiwillen, sondern wurzelt in dem Gehalt der Regeln selbst. Der Grund für die Bindung der Parteien an die Regeln liegt danach in deren Effizienz oder Fairness oder Richtigkeit oder welches substanzielle Kriterium auch immer zu Grunde gelegt wird. Während die erste Rechtfertigungsmöglichkeit prozedural oder vom »Locke«-Typus ist (man ist nur gebunden, wenn man sich freiwillig für das Geschäft entschieden hat), ist die zweite materiell oder vom »Hobbes«-Typus (man ist gebunden, weil es sich um die bestmögliche Art von Geschäft handelt, unabhängig davon, ob man es frei gewählt hat). Während die erste Rechtfertigungsmöglichkeit auf internationale Handelsgeschäfte passt, bei denen die Parteien gezielt eine bestimmte Rechtswahl treffen, ${ }^{15}$ scheint die zweite Rechtfertigungsmöglichkeit besser auf Massengeschäfte mit Verbrauchern zu passen, für die ein zwingendes Verbraucherschutzrecht gilt. Während die erste Variante durch und durch vertragsrechtlich ist, trägt die zweite die Maske des Vertrags, aber gehört in Wahrheit zu einem anderen Zweig des Schuldrechts.

Drittens schließlich wende ich mich den verschiedenen Anwendungsmöglichkeiten des Netzwerk-Konzepts zu. Eine typisch vertragsrechtliche Anwendung besteht darin, dass die Parteien sich gezielt für das Netzwerk als Vertragsregime entscheiden können. Eine zweite Anwendung besteht darin, einen Komplex von Transaktionen unter ein Netzwerk-Regime zu stellen, nicht weil die Parteien dies gewollt hätten, sondern weil es sich materiell-inhaltlich rechtfertigen lässt. Schließlich könnte das NetzwerkKonzept aber auch als integraler Bestandteil des englischen Vertragsrechts begriffen werden, unabhängig davon, ob die Netzwerk-Regeln frei gewählt oder auferlegt sind. Es ist vielleicht diese dritte Art der Anwendung, mit der am besten auf das dogmatische Problem im ersten Teil des Aufsatzes reagiert werden kann. Die größere allgemeine Bedeutung kommt freilich den beiden ersten Anwendungsmöglichkeiten zu.

\section{Die anhaltende Relevanz von Netzwerken im Vertragsrecht}

Unter der Rechtslage nach dem Contracts Rights of Third Parties Act 1999 können die Parteien eines Vertrages, A und B, vereinbaren, dass eine dritte Partei, C, ein eigenes

14 Vgl. D.R. Johnson und D. Post (1996) »Law and Borders - The Rise of Law in Cyberspace«, 48 Stanford Law Review, 1367-1402.

15 Vgl. Gunther Teubner (2002) »Breaking Frames: Economic Globalisation and the Emergence of the Lex Mercatoria«, 5 European Journal of Social Theory, 199-217. 
Recht zur Durchsetzung des zwischen ihnen geschlossenen Vertrages erhalten soll. ${ }^{16}$ Ebenso wird C zur Durchsetzung des Vertrages zwischen A und B berechtigt sein, wenn es die Intention der Vertragsparteien ist, durch ihre Vereinbarung $\mathrm{C}$ zu begünstigen; dies gilt jedenfalls dann, wenn sie nicht beabsichtigen, dem $\mathrm{C}$ das Recht zur Durchsetzung des Vertrages vorzuenthalten. ${ }^{17}$ Bei Anwendung dieser Vorschriften sollte es möglich sein, gravierende Ungerechtigkeiten zu vermeiden, insbesondere in Fällen, in denen Familienangehörige die begünstigten Dritten sind wie in Tweedle $v$ Atkinson $^{18}$ und Beswick v Beswick. ${ }^{19}$

Während das Gesetz das einfachere Problem eines begünstigten Dritten regelt, behandelt es ausgerechnet jene Fälle in zweideutiger Weise, die den Gerichten in der Hochphase der Überlegungen zur Reform des Relativitätsprinzips das meiste Kopfzerbrechen bereiteten. In diesen Fällen schlossen A und B den Hauptvertrag und B sodann einen Untervertrag mit C (zum Beispiel könnte B ein Spediteur sein, der die Arbeit des Verladens an $\mathrm{C}$ vergibt, oder ein Bauunternehmen, dass einen Untervertrag über die Elektroarbeiten mit C schließt). Hier war es Gang und Gäbe, dass B sich in seinem Vertrag mit A über eine Haftungsbegrenzung einigte, die nicht nur ihn selbst, sondern alle nachfolgenden Parteien von Unterverträgen (wie C) von der Haftung freistellte - das war die von sog. »Himalajaklauseln« beabsichtigte Wirkung. Obgleich A nicht direkt gegen $\mathrm{C}$ aus Vertrag vorgehen kann, könnte er auf die Idee kommen, C wegen fahrlässigem unerlaubtem Handeln zu verklagen (typischerweise dann, wenn $C$ unachtsam das Eigentum des A beschädigt). Wenn $C$ sich nun auf eine Haftungsbeschränkung berufen würde, die in dem Hauptvertrag zwischen A und B oder in dem Untervertrag zwischen B und C vereinbart worden ist, so müsste eine solche Verteidigung eigentlich an einer strikten Anwendung des Relativitätsprinzips scheitern. ${ }^{20}$ Der Third Parties Act 1999 wird dem Schwierigkeitsgrad dieser Fälle kaum gerecht; es sieht nur vor, dass die Lockerung des Relativitätsprinzips dem Dritten erlauben soll, sich ebenso wie auf die Vergünstigung in dem Vertrag zwischen A und B auch auf eine etwa vorhandene Einwendung zu berufen. ${ }^{21}$

Unter einem gelockerten Relativitätsprinzip gibt es eigentlich keinen Grund, warum es einem Subunternehmer wie C verwehrt sein sollte, sich auf einen Haftungsausschluss im Vertrag zwischen A und B zu berufen, um einem Anspruch des A entgegenzutreten. Wie aber sieht es aus, wenn A als klagende Partei die Angemessenheit des Haftungsausschlusses in Frage stellt? A bezweifelt in einem solchen Fall nicht, dass sich $\mathrm{C}$ nicht auf die Klausel berufen könne; dieses Argument ist von der neuen Gesetzgebung abgeschnitten. Vielmehr macht A geltend, dass die Klausel inhaltlich unangemessen sei - einen Einwand, den A nach Erlass des Unfair Contract Terms Act 1977 nicht nur als Verbraucher, sondern auch als Unternehmer erheben kann. Das Problem entsteht, weil A den Einwand gegenüber $\mathrm{C}$ als einer Person erhebt, mit der er

$16 \S 1(1)(a)$.

$17 \S 1(1)(b)$ und $\S 1(2)$.

18 [1861] 1 B\&S 393.

19 [1968] AC 58.

20 Vgl. Scruttons Ltd v Midland Silicones Ltd [1962] AC 446.

$21 \S 1(6)$. 
keine direkte Vertragsbeziehung hat, und gerade nicht gegenüber B, mit dem er in einer vertraglichen Beziehung steht, die als solche ohne weiteres im Hinblick auf ihre inhaltliche Fairness beurteilt werden kann. Anders ausgedrückt, liegt das Problem nicht einfach darin, dass das, was zwischen A und B angemessen ist, nicht automatisch auch zwischen $\mathrm{A}$ und $\mathrm{C}$ angemessen ist, sondern es ist überhaupt unklar, anhand welcher Referenz die Angemessenheit im Verhältnis zwischen A und C zu beurteilen ist.

Im Standardfall, in dem zwischen A und B ein Vertrag besteht und A die Angemessenheit einer Klausel bestreitet, auf die sich B beruft, würde die Angemessenheit anhand der Geschäftsbeziehung zwischen A und B beurteilt werden. ${ }^{22}$ Die Gerichte würden die Klausel überprüfen mit Blick auf verschiedene Aspekte wie zum Beispiel der relativen Verhandlungsmacht der Parteien im Verhältnis zueinander, dem Umstand einer möglichen Bestimmung der einen durch die andere Partei oder sie würden danach fragen, wer von beiden, A oder B, sich besser gegen Risiken versichern könnte. Die Beurteilung solcher Aspekte folgt keinen wissenschaftlich exakten Regeln. Es gibt keine Skala, mit deren Hilfe die Verhandlungsmacht einer Partei gemessen werden könnte: Wir können nicht sagen, A erreiche einen Wert von 5 auf dieser Skala und B einen Wert von 7; alles, was wir sagen können, ist, dass B über eine größere Verhandlungsstärke verfügt als A. Vor allem besagt der Umstand, dass eine Klausel unter dem Strich angemessen im Verhältnis zwischen A und B ist, noch lange nicht, dass die Klausel grundsätzlich als Inbegriff eines fairen Interessenausgleichs dienen kann; gesagt ist damit lediglich, dass die Klausel nicht als offensichtlich unangemessen verworfen werden darf und im Rahmen eines Spielraums akzeptiert werden kann. Weil vergleichsweise wenig Fälle unangemessener Vertragsklauseln berichtet werden, lässt sich zudem schwer sagen, wie breit der Spielraum eigentlich ist. Mit einiger Sicherheit steht wohl nur fest, dass der Spielraum bei Verträgen zwischen Unternehmern größer ist als bei Verbraucherverträgen. ${ }^{23}$ Welchen Umfang der Spielraum aber auch konkret haben mag, im vorliegenden Zusammenhang ist allein wichtig, dass es die geschäftlichen Beziehungen zwischen A und B sind, die den Referenzrahmen für die Beurteilung der Angemessenheit abgeben, oder, um es in der Sprache von § 11(1) UCTA zu sagen: die Frage ist, ob eine Klausel »angemessen [ist] im Hinblick auf die Umstände, die die Parteien kannten oder hätten kennen müssen oder sich vorgestellt haben, als sie den Vertrag schlossen ${ }^{24}$

22 Das gleiche würde gelten, wenn sich die Angemessenheitsprüfung darauf bezöge, ob die sich auf die Klausel berufende Partei in gutem Glauben gehandelt hätte.

23 Vgl. John Adams und Roger Brownsword (1988) »The Unfair Contract Terms Act: A Decade of Discretion «, 104 Law Quarterly Review, 94 ff. Der Spielraum bei Handelsverträgen wird deutlich in Fällen wie Photo Production Ltd v Securior Transport Ltd [1980] AC 827 und, aus jüngster Zeit, Watford Electronics Ltd v Sanderson CFL Ltd [2001] EWCA Civ 317.

24 Dieser Test, der die Vernünftigkeit der Klausel zum Zeitpunkt des Vertragsschlusses beurteilt, wurde einem Test vorgezogen, der hierfür auf den Zeitpunkt des Konflikts abstellt. Selbst wenn man sich für letzteres entschieden hätte, bliebe das im Text beschriebene Problem bestehen. 
Das erwähnte Problem entsteht jedoch gerade, wenn es $\mathrm{C}$, und nicht $\mathrm{B}$, ist, der sich auf eine bestimmte Ausschlussklausel beruft. Die naheliegende Frage ist dann folgende: Sind es immer noch die Geschäftsbeziehungen zwischen A und B, die den Rahmen für die Beurteilung der Angemessenheit der Klausel abgeben? Oder sollte nicht vielmehr der Referenzrahmen gewechselt und B gegen $\mathrm{C}$ ausgetauscht werden, obwohl A keinen direkten Geschäftskontakt mit $\mathrm{C}$ hat? Der Gesetzgeber gibt uns keine Handreiche für die Lösung solcher Streitfälle.

Bevor wir unsere Überlegungen zum richtigen Prüfungsmaßstab in solchen Fällen fortsetzen können, muss freilich noch ein technischer Aspekt klargestellt werden. Gemäß $§(2)$ des Gesetzes von 1999 gilt:

$\S 2$ (2) des Unfair Contract Terms Act 1977 (über den Ausschluss einer Haftung für Fahrlässigkeit usw.) bleibt unangewendet, wenn die Fahrlässigkeit in der Verletzung einer vertraglichen Verpflichtung besteht und der sich hierauf Berufende ein Dritter nach $\S 1$ ist.

Obwohl diese Vorschrift sich auf das Zusammentreffen der modifizierten Relativitätsregel und dem UCTA bezieht, lässt sich aus ihr nichts für unseren Problemfall gewinnen. Um dies zu verstehen, müssen wir den Bedingungssatz der Vorschrift näher beleuchten, nach dem die Person, die sich auf die Klausel beruft, ein Dritter ist, der sich auf die Vorschriften zur Durchsetzbarkeit in dem Gesetz von 1999 stützt.

Nehmen wir zunächst an, dass A den Vertrag zwischen B und C unter Berufung auf die Vorschriften des Gesetzes von 1999 durchzusetzen versucht und behauptet, C habe die vertraglichen Sorgfaltspflichten verletzt (C habe fahrlässig gehandelt). Nehmen wir weiter an, dass B und $\mathrm{C}$ die Anwendbarkeit dieser Vorschriften nicht bestreiten, weil es ihre Absicht war, einen Vertrag zugunsten von A zu schließen, und setzen wir ebenfalls voraus, dass $\mathrm{C}$ das ihm von A vorgeworfene Verhalten einräumt. Die Vorschrift des § 1(4) [sic] des Gesetzes von 1999 bewirkt dann, dass A in gleicher Weise in den Vertrag einbezogen wird wie B; woraus wiederum folgt, dass A an mögliche Klauseln zur Begrenzung der Haftung (des C) in dem Vertrag zwischen B und C gebunden ist. Sofern A die Angemessenheit solcher Klauseln in Frage zu stellen beabsichtigt, wird er daran durch $\S 7(2)$ des Gesetzes von 1999 gehindert, der eine Berufung auf $\S 2(2)$ des UCTA und damit auf jene Vorschrift abschneidet, auf die anderenfalls eine Überprüfung der Angemessenheit einer vertraglichen Beschränkung der Haftung des C (ausgenommen die fahrlässige Verletzung von Leib oder Leben) gestützt werden könnte. ${ }^{25}$

Im Gegensatz dazu will der A in dem vorliegend relevanten Szenario, das die Gerichte im Vorfeld des Gesetzes von 1999 beschäftigte, den C direkt für dessen Fahrlässigkeit in Anspruch nehmen. Das Klagerecht des A fußt nicht auf dem Gesetz von 1999, so dass entsprechend $§ 7(2)$ nicht anwendbar ist. In einem solchen Fall könnte sich C auf eine Haftungsbeschränkung (i) aus dem Vertrag zwischen A und B oder (ii) aus dem Vertrag zwischen B und C zu berufen suchen. Dann erlaubt das Gesetz von 1999 dem C zwar, sich auf ersteren zu berufen (in Übereinstimmung mit den Absich-

25 Wenn durch die Fahrlässigkeit Leib oder Leben verletzt werden, schließt § 2(1) UCTA die Berufung auf jegliche haftungseinschränkende Klausel aus. 
ten von A und B), aber es hat keine Wirkung in Bezug auf letzteren, so dass diese Konstellation für das Common Law weiterhin schwierig bleibt. ${ }^{26} \mathrm{Zu}$ unserem Testfall wird folglich ein solcher, in dem A den C ohne Berufung auf das Gesetz von 1999 wegen Fahrlässigkeit verklagt und $\mathrm{C}$ sich auf eine Haftungsbeschränkung in dem Vertrag zwischen A und B beruft, die zum Vorteil des C aufgestellt wurde. Einmal mehr stellt sich dann die Frage: Macht es Sinn, wenn ein Gericht die Angemessenheit einer haftungsbeschränkenden Klausel, die im Mittelpunkt eines Streits zwischen A und C steht, im Hinblick auf die Geschäftsbeziehungen zwischen A und B beurteilt?

Im Folgenden wollen wir zu Illustrationszwecken Verträge aus dem Baugewerbe unter dem Aspekt unterschiedlicher Verhandlungsstärke der beteiligten Parteien betrachten. Im Grundfall eines Konflikts zwischen A (dem Kunden) und B (dem Hauptunternehmer) hat das Gericht zu ermitteln, ob zwischen A und B ein Verhandlungsungleichgewicht bestanden hat, und ob gegebenenfalls die überlegene Partei ihre Verhandlungsmacht unzulässig ausgenutzt hat. Wenn der Streit zwischen B (dem Hauptunternehmer) und C (einem Subunternehmer) ausbricht, wird das Gericht ganz ähnlich vorgehen, wobei jetzt natürlich die Geschäftsbeziehung zwischen B und C einschlägig ist. Wenn aber der Konflikt zwischen A und C entsteht, wie fließt dann die relative Verhandlungsstärke der Parteien in das Urteil über die Angemessenheit der Klausel ein, auf die C sich stützen will?

Wenn wir die relative Verhandlungsstärke aller drei beteiligten Parteien (A und B, B und $\mathrm{C}$ sowie $\mathrm{A}$ und $\mathrm{C}$ ) berücksichtigen, so ergeben sich sechs mögliche Varianten (genauer drei Paare von Varianten, abhängig davon, ob es $\mathrm{A}, \mathrm{B}$ oder $\mathrm{C}$ ist, der über die größte Verhandlungsmacht verfügt). Da es allein um den einen Fall geht, dass A die Angemessenheit der Klausel bestreitet, auf die sich $\mathrm{C}$ stützt, vereinfachen sich die Möglichkeiten auf folgende zwei Varianten:

(V1) $(\mathrm{A}>\mathrm{B}>\mathrm{C} ; \mathrm{B}>\mathrm{A}>\mathrm{C}$; und $\mathrm{B}>\mathrm{C}>\mathrm{A}) \mathrm{A}$ ist in einer stärkeren Verhandlungsposition im Verhältnis zu $C$ als im Verhältnis zu $B$;

(V2) $(\mathrm{A}>\mathrm{C}>\mathrm{B} ; \mathrm{C}>\mathrm{B}>\mathrm{A}$; und $\mathrm{C}>\mathrm{A}>\mathrm{B}) \mathrm{A}$ ist in einer stärkeren Verhandlungsposition im Verhältnis zu B als im Verhältnis zu $C$.

In Variante V1 wird A argumentieren, dass C übermäßig geschützt ist, weil A bei direkten Verhandlungen mit $\mathrm{C}$ weniger Zugeständnisse gemacht hätte (d.h. weniger als A dem B gegenüber eingeräumt hat); die Haftungsbeschränkung hätte nicht den gleichen Umfang gehabt. Nach Ansicht von A darf das Gericht, selbst wenn es die Klausel im Hinblick auf die Verhandlungen zwischen A und B für angemessen halten würde, dies nicht auch im Verhältnis zu C tun. Mit anderen Worten behauptet A, dass es ungerecht sei, wenn $\mathrm{C}$ von einem Zugeständnis aus dem Vertrag zwischen A und B profitiere, obwohl A in Verhandlungen mit $\mathrm{C}$ das gleiche Zugeständnis nicht gemacht hätte.

In Variante V2 scheint A kaum auf das Argument aus V1 zurückgreifen zu können: denn wenn A dem B eine gewisses Maß an Haftungsbeschränkung gewährt hat, kann er nicht plausibel behaupten, er hätte eine für sich selbst günstigere Klausel mit $\mathrm{C}$ vereinbart, der doch ihm gegenüber - so die Prämisse - die größere Verhandlungsstärke

26 Vgl. Morris v C.W. Martin and Sons Ltd [1966] 1 QB 716. 
besitzt. Vielmehr müsste A argumentieren, dass es von $\mathrm{C}$ unfair sei, von einer Haftungsbeschränkung zu profitieren, die A dem B als Gegenopfer gewährt hat, während C sich (als stärkere Verhandlungspartei) nicht auf ein solches (zu geringes) Zugeständnis eingelassen hätte. Mit anderen Worten behauptet $\mathrm{A}$, dass es ungerecht sei, wenn $\mathrm{C}$ von einem Zugeständnis aus dem Vertrag zwischen A und B profitiert, obwohl C in Verhandlungen mit A zu keinerlei eigenem Opfer bereit gewesen wäre.

Das Argument von A ist in mehrfacher Hinsicht angreifbar. Zum einen scheint sich A zu widersprechen, wenn er sich auf seine relative Verhandlungsstärke (in V1) und gleichzeitig auf seine relative Unterlegenheit (in V2) beruft, um die Angemessenheit der Klausel zu begründen. Zudem setzt das Argument als selbstverständlich voraus, dass die Parteien rein antagonistisch verhandeln, was unter einer Theorie relationaler Verträge als fragwürdig erscheint. Gleichwohl steckt hinter der Argumentation von A ein berechtigtes Anliegen. Das Gericht ist aufgefordert, den Referenzrahmen für Angemessenheitsprüfung zu suchen, wenn es $\mathrm{C}$ und nicht B ist, der sich auf die betreffende Klausel beruft. Die Schwierigkeit besteht darin, dass es keine direkte Geschäftsbeziehung zwischen $\mathrm{A}$ und $\mathrm{C}$ gibt, auf die ein solcher Maßstab gegründet werden könnte.

Wir wechseln nun den Kontext und gehen über von Handels- zu Verbraucherverträgen und vom Bau- zum Reinigungsgewerbe. Angenommen, A ist ein Verbraucher, der regelmäßig B, ein Reinigungsunternehmen, beauftragt; B selbst lässt die Arbeiten oft von Subunternehmern, unter anderen von C, ausführen. Zu unserem Testfall kommt es dann, wenn $\mathrm{C}$ das Eigentum des A beschädigt, A daraufhin $\mathrm{C}$ für dessen Fahrlässigkeit verklagt, und sich $\mathrm{C}$ verteidigt, indem er sich auf Schutzklauseln aus dem Vertrag zwischen A und B beruft. Angenommen, B hat im konkreten Fall dem A eine besondere Vergünstigung (z.B. einen erheblichen Nachlass auf den üblichen Preis) im Austausch für eine weitergehende Beschränkung der Haftung angeboten. A hält es als für angemessen, wenn sich B selbst auf diese spezielle Beschränkung berufen kann, akzeptiert jedoch nicht, dass $\mathrm{C}$ in gleicher Weise hiervon profitieren soll. Oder gesetzt den anderen Fall, dass B den A erst nach Vertragsschluss über die Vertragsbedingungen unterrichtet, gleichwohl aber die von B gestellten Klauseln aufgrund des Prinzips der »vorausgegangenen Geschäfte « wirksam einbezogen werden. ${ }^{27}$ Auch hier gesteht A zu, dass die von B aufgestellten Klauseln zu dessen, nicht aber zu C's Gunsten wirksam einbezogen worden sind. Wie in dem Bauprojekt-Fall argumentiert A, dass das, was zwischen A und B angemessen sei, nicht notwendig auf das Verhältnis zwischen A und $\mathrm{C}$ übertragbar sei. Wiederum liegt das Problem nicht einfach darin, dass sich die inter-personell ausgehandelte Fairness nicht übertragen lässt, sondern darin, dass überhaupt nicht klar ist, welcher Maßstab hier einschlägig ist.

Eine andere Entwicklung im englischen Vertragsrecht aus jüngerer Zeit lässt das Problem noch deutlicher hervortreten. Es handelt sich um einen von Lord Hoffmann in Investors Compensation Scheme Ltd $v$ West Bromwich Building Society ${ }^{28}$ vertretenen

27 Vgl. etwa McCutcheon v David MacBrayne Ltd [1964] 1 All ER 430.

28 [1988] 1 All ER 98. Ähnlich Lord Hoffmann wie auch Lord Steyn in Mannai Investments Co Ltd v Eagle Star Life Assurance Co Ltd [1997] 3 All ER 352. 
neuen, kontextbezogenen Ansatz zur Interpretation von Handelsverträgen. Nach Ansicht von Lord Hoffmann ist »beinahe alles alte intellektuelle Gepäck der ,rechtlichen' Interpretation abgeworfen $\ll^{29}$, speziell der abstrakte »Literalismus «. Während sich letzterer der einfachen, natürlichen und gewöhnlichen Bedeutung der von den Vertragsparteien verwendeten Sprache verschrieben habe, sucht der neue Kontextualismus »die Bedeutung [zu ermitteln], die das Dokument für eine vernünftige Person mit dem ganzen Hintergrundwissen, das auch den Vertragsparteien im Zeitpunkt des Vertragsschlusses vernünftiger Weise zur Verfügung stand, besitzen würde $\ll{ }^{30}$ Kontextualistische Interpretation baut demnach auf drei Elementen auf: die Bedeutung eines Vertrages ist zu ermitteln (i) aus Sicht einer vernünftigen Person, (ii) die sich in der Situation der Parteien zum Zeitpunkt des Vertragsschlusses befindet und (iii) die über das den Parteien zu dieser Zeit mögliche Hintergrundwissen verfügt. Wie sich aus diesen drei Elementen klar ergibt, sind es die Geschäfte zwischen den konkreten Vertragsparteien (A und B), die den unmittelbaren Kontext für die Interpretation bilden.

Seit der Entscheidung Investors ist deutlich, dass selbst die einfach erscheinende Konstellation zwischen A und B sich mitunter einer schnellen kontextuellen Auflösung entziehen kann; ${ }^{31}$ wobei aber immerhin der Kontext, nämlich die Geschäftsbeziehungen zwischen A und B, feststeht. Schwieriger wird es, sofern C mit ins Spiel kommt und eine Klausel aus dem Vertrag zwischen A und B zu seinen Gunsten in Anspruch nehmen will, die Klausel jedoch anders auslegt als A oder B. Welcher Bezugsrahmen ist hier anzulegen?

Das Problem sollte in seiner allgemeinen Form deutlich geworden sein. Im einfach gelagerten Ausgangsfall macht es Sinn, vertragliche Streitigkeiten unter Berücksichtigung aller geschäftlichen Kontakte zwischen den Parteien zu lösen. Sobald eine dritte Partei mit ins Spiel kommt, fehlt es an einem evidenten Bezugsrahmen. Wenn wir uns auf die Geschäfte zwischen A und B und den von beiden ausgehandelten Vertrag beziehen, um eine Streitigkeit zwischen A und C zu lösen, so wird die Entscheidung offensichtlich anhand eines falschen Maßstabs getroffen. Wenn wir andererseits versuchen, geschäftliche Kontakte zwischen A und C zu konstruieren, so versteigen wir uns zu einer Fiktion. An diesem Punkt bietet sich das Netzwerk als besserer Lösungsansatz an, der den Erwartungen der Parteien Rechnung trägt. Denn der Netzwerk-Ansatz zwingt zu keiner Entscheidung zwischen zwei verschiedenen, voneinander getrennt zu behandelnden Sets von Geschäften. Stattdessen scheint er die Behandlung der Frage in einem Zusammenhang zu ermöglichen, in dem die Transaktionsumgebung, in der sich die Parteien tatsächlich befinden, besser abgebildet wird. Außerdem verspricht der Ansatz Lösungen, die eher den vernünftigen Erwartungen der Vertragsparteien entsprechen.

29 Hoffmann (Fn. 27) 114.

30 Hoffmann (Fn. 27) 114.

31 Vgl. Roger Brownsword (2003) ^After Investors: Interpretation, Expectation and the Implicit Dimension of the ,New Contextualism' «, in: David Campbell, Hugh Collins und John Wightman (Hg.) The Implicit Dimensions of Contract, Oxford: Hart, 103-142. 
In diesen Überlegungen deutet sich die Fruchtbarkeit der Idee von Netzwerk-Verträgen an. Um jedoch ihre Bedeutung vollständig zu erfassen, müssen wir einen Schritt zurückgehen und Licht in zwei unterschiedliche Begründungsansätze bringen - in zwei Wege, die Durchsetzung der Erwartungen einer Vertragspartei zu rechtfertigen. $\mathrm{Zu}$ unserer Überraschung werden wir feststellen, dass die Durchsetzung von Verpflichtungen unter einem Netzwerk-Regime in Wahrheit der idealtypische Fall einer vertraglichen Verpflichtung ist und nicht etwa einen entlegenen Sonderfall oder eine Ausnahme darstellt.

\section{Zwei Begründungsansätze}

Hat A einen Vertrag mit B und B seinerseits einen Vertrag mit C geschlossen, so stoBen wir auf Schwierigkeiten, sofern (i) die Dogmatik des Vertragsrechts einen einzigen Vertrag als Referenzrahmen für vertragliche Streitigkeiten verwendet und (ii) eine vertragliche Streitigkeit zwischen A und C entsteht. Es ist nicht einsichtig, warum ein solcher Disput, zwischen A und C, unter Heranziehung der ganz anderen geschäftlichen Verbindung zwischen A und B (oder der Geschäfte zwischen B und C) beurteilt werden sollte; noch ist klar, wie sich eine vertragliche Beziehung zwischen A und C begründen ließe. Wie erwähnt, könnte der Netzwerk-Gedanke die Gerichte in die Lage versetzen, die Streitigkeit zwischen A und C in einem adäquateren Rahmen zu behandeln, wobei bisher offen geblieben ist, wie dies zu bewerkstelligen ist. In diesem Abschnitt möchte ich zwei unterschiedliche Lösungsstrategien vorstellen, wobei stets die vernünftigen Erwartungen der Parteien im Auge zu behalten sind. Eine dieser Strategien basiert auf einer prozeduralen Rechtfertigung; auf dem Umstand, dass die Parteien sich freiwillig ein rechtliches Regime gegeben haben. Die andere Strategie fußt auf einer materiell-inhaltlichen Rechtfertigung; also nicht auf dem Umstand, dass die Parteien sich selbst ein rechtliches Regime gegeben haben, sondern darauf, dass diese Regeln die bestmöglichen für die konkreten Zwecke der Parteien sind.

Um mit den Grundlagen zu beginnen: A, B und C benötigen nicht zwangsläufig ein dogmatisches System formeller Rechtsregeln, die zusammengenommen ein »Schuldrecht« bilden, um miteinander Geschäfte zu tätigen. Ohne Zugriff auf ein System des Vertragsrechts würden Unternehmer freilich dazu tendieren, weniger Risiken zu übernehmen; und Verbraucher hätten im Konfliktfall noch weniger sicheren Boden unter den Füßen, auf dem sie tätig werden könnten. Grob gesprochen, lässt sich sagen, dass ein System des Vertragsrechts den Parteien (durchsetzbare) Rechte gewährt, die anderenfalls nicht bestehen würden, und dass es die Parteien ermutigt, sich auf geschäftliche Beziehungen auch jenseits von konkreten Vertrauensverhältnissen einzulassen. Auch wenn wir ein formales System des Vertragsrechts auf Märkten als selbstverständlich gegeben voraussetzen, sollten wir uns in Erinnerung rufen, dass Märkte auch ohne ein Vertragsrecht funktionieren können.

Grundsätzlich gibt es zwei Möglichkeiten, die Anwendbarkeit von Vertragsrecht auf geschäftliche Beziehungen zu begründen. Erstens können A und B vereinbaren, dass ihre Geschäfte dem Vertragsrecht unterfallen sollen. Einigen sie sich hierauf, so können sie sich später nicht über die im Konfliktfall konkret anzuwendenden Regeln des 
Vertragsrechts beklagen. Die einschlägigen Regeln mögen schmerzhaft oder gar unfair sein; wenn aber die Parteien ihrer Anwendung vorab zugestimmt haben, gibt es keinen Grund zur Beschwerde. Wir haben es mit einer prozeduralen Begründung zu tun. Zweitens kann es sein, dass A und B sich keinerlei Gedanken darüber gemacht haben, ob ihre Geschäftsbeziehung dem Vertragsrecht unterworfen sein soll, der Staat jedoch verfügt hat, dass Geschäfte wie die zwischen A und B dem Vertragsrecht unterfallen sollen. Wenn etwa in unserem Beispielsfall der Verbraucher A seine Kleidung in die Reinigung des B bringt, wird er kaum über die Anwendung des Vertragsrechts auf seine geschäftlichen Beziehungen zu B nachgedacht haben. Dennoch steht fest, dass Vertragsrecht Anwendung findet. Sofern das anwendbare Recht für A und B zwingend ist, muss die Begründung für seine Anwendung eine materiell-inhaltliche sein; der Staat muss die anwendbare Rechtsregel inhaltlich rechtfertigen können (entweder unter Bezug auf eine Theorie der Gerechtigkeit oder unter Hinweis auf die Effizienz der Regel oder auf irgendeine andere Art). Sofern hingegen A und B das anwendbare Recht abbedingen dürfen (im englischen Recht dadurch, dass sie erklären, kein Rechtsverhältnis eingehen zu wollen), ${ }^{32}$ und wenn A und B von einem solchen »opt-out« keinen Gebrauch machen, erscheint eine prozedurale Begründung weiter möglich. Indessen ist ein solcher Ansatz auf Märkten mit Massenverträgen unter Verbrauchern unrealistisch. Die Parteien sind sich größtenteils nicht bewusst, dass sie dem Vertragsrecht unterworfen sind, und noch viel weniger, dass eine Möglichkeit des »opting out « besteht. Dem entsprechend ist immer dann, wenn die Parteien dem Vertragsrecht aus Zweckmäßigkeitserwägungen zwingend unterworfen werden und es von ihnen nicht frei vereinbart werden kann, eine materiell-inhaltliche Begründung notwendig.

Angesichts der Bedeutung, die die prozedurale Begründungsvariante für den vorliegend vertretenen Ansatz besitzt - für die Idee, dass Vertragsparteien unter Umständen gut beraten sind, ihre Geschäfte bewusst unter ein Netzwerk bzw. Netzwerk-Regime zu stellen -, sind einige Worte zur Natur dieser Begründungsstrategie angebracht. Im idealen Fall werden sich A und B, bevor sie in geschäftliche Beziehungen zueinander treten (bevor A beginnt, mit B über die geplanten Baukonstruktionen zu verhandeln), über jenes Set von Regeln einigen, das auf ihre Geschäftsbeziehung anwendbar sein soll. Mit anderen Worten, es ist eine Vorvereinbarung erforderlich, in der die Regeln festgelegt werden, denen das eigentliche Rechtsgeschäft unterfallen soll. Damit ein infiniter Regress vermieden wird, weil eine Vorvereinbarung den Hauptvertrag und eine Vor-Vorvereinbarung die Vorvereinbarung abdecken muss und so fort, sind ein paar zwingende Grundregeln erforderlich, die für die Vorvereinbarung gelten. Die Rechtsdogmatik muss angeben können, unter welchen Bedingungen wir von einer wirksamen (autorisierenden) Vorvereinbarung auszugehen bereit sind. Je nachdem, welches theoretische Konzept von Konsens, von Stellvertretung usw. vertreten wird, ergeben sich jeweils andere spezifizierende Aussagen darüber, wann die Parteien eine freie und informierte Wahl treffen, wo die Schwelle für Geschäftsfähigkeit anzusetzen ist, wie

32 Vgl. Rose and Frank Company v J.R. Crompton and Brothers Ltd [1925] AC 445. 
Auslegungsschwierigkeiten gelöst werden, usw. Das alles sind schwierige Fragen; im vorliegenden Zusammenhang reicht es aus, auf ihre Existenz hinzuweisen. ${ }^{33}$

Lassen wir deshalb jede Komplikation beiseite und nehmen an, dass die Dogmatik der Vorvereinbarungen umfassend entwickelt ist. Der Staat mag einzelnen privaten Normsetzungsinstitutionen Grenzen ziehen ${ }^{34}$ innerhalb dieser aber sind die Parteien darin frei, ihre Geschäfte beliebigen Regeln zu unterstellen. Es kann sich dabei um das lokale Vertragsrecht oder das eines ganzen Rechtsraumes (wie z.B. das europäische) handeln, aber ebenso um ein Set informeller Regeln oder die Regeln einer bestimmten Branche für bestimmte Leistungsmodalitäten (z.B. bei Benutzung elektronischer Frachtbriefe). Welche Regeln auch immer von der Vorvereinbarung für anwendbar erklärt werden, stets ist ihre Anwendung in diesen Fällen prozedural gerechtfertigt.

Würde ein bestimmtes, auf die Regulierung von Transaktionen im Netzwerk zugeschnittenes Set von Regeln entwickelt, so hätten die Geschäftspartner auch die Möglichkeit, auf diese Regeln zurückzugreifen. Mit anderen Worten, A und B würden übereinkommen, ihre Geschäfte den »Regeln für Netzwerkverträge« zu unterstellen; weder A noch B hätten dann einen Grund, sich zu beklagen, wenn diese Regeln dann auch tatsächlich angewendet würden. Freilich gehört es zu den Eigenschaften des prozeduralen Begründungsansatzes, dass er nur zwischen den Parteien der Vorvereinbarung trägt. Weder A noch B können sich gegen die Anwendung der Netzwerk-Regeln wenden. Aber diese binden nicht den $\mathrm{C}$ oder irgend eine andere dritte Partei. Damit der prozedurale Begründungsansatz auch auf Dritte erstreckt werden kann, müssen diese sich ebenfalls der Vorvereinbarung unterwerfen und die eigenen Geschäfte von dem gewählten Rechtsregime regeln lassen. Auf dieser Grundlage lassen sich zwei schwierige Fragen im Zusammenhang mit dem Netzwerk-Gedanken beantworten, nämlich wie Mitglieder des Netzwerks von externen Parteien unterschieden und wie die Beziehungen zwischen beiden geregelt werden können. Wenn auf die prozedurale Begründung für die Anwendbarkeit von Netzwerk-Regeln zurückgegriffen werden kann, dann sind Mitglieder solche und nur solche Personen, die sich der Vorvereinbarung unterstellt haben; und wenn die Anwendung von Netzwerk-Regeln auf externe Parteien in Betracht gezogen wird, dann muss die Begründung hierfür eine materiell-inhaltliche anstatt einer prozeduralen sein.

Als nächstes müssen wir uns vergegenwärtigen, dass die Zustimmung zu den Klauseln einer Vorvereinbarung von der Zustimmung zum Hauptvertrag verschieden ist und dieser vorausgeht. Es ist allgemein anerkannt, dass eine vertragliche Verpflichtung auf Zustimmung beruht und dass es der Konsens der Beteiligten ist, der eine vertragliche Verpflichtung von Pflichten aus Delikt, Eigentum, Bereicherung oder sonstigen Rechtsgründen unterscheidet. ${ }^{35}$ Entgegen der allgemeinen Meinung ist aber nicht

33 Umfassend vgl. Deryck Beyleveld und Roger Brownsword (2006) Consent in the Law, Oxford: Hart.

34 Bei der Durchsetzung der gewählten Regeln mit Hilfe staatlicher Gerichte kann es zu Vorbehalten gegenüber Regelsets kommen, die sich nicht auf eine formale Rechtsquelle zurückführen lassen. Vgl. Roy Goode (1997) »Usage and its Reception in Transnational Commercial Law «, 46 ICQL, 1-36.

35 Vgl. etwa Hein Kötz und Axel Flessner (1998) European Contract Law (Volume One), Oxford: Clarendon. 
die Zustimmung zum Hauptvertrag (das Phänomen des Übereinkommens bzw. Angebot und Annahme) der Schlüssel zur Begründung einer vertraglichen Verpflichtung; vielmehr ist es die Zustimmung zur Vorvereinbarung des Rechtsregimes, die die tiefere und eigentliche Quelle für die vertragliche Bindung ist. Ein Vertrag realisiert sich in den folgenden vier Schritten:

(i) A und B treffen eine Vorvereinbarung, nach der ihre Geschäfte etwa dem »englischen Vertragsrecht « unterstellt werden sollen;

(ii) Aufgrund ihrer Zustimmung zu den Klauseln der Vorvereinbarung haben A und B die Regeln des englischen Vertragsrechts als Rechtsregime für ihre Transaktionen angenommen;

(iii) Gemäß dem anwendbaren Recht (auf das sich A und B aus freien Stücken geeinigt haben) wird eine Transaktion nur dann als rechtlich durchsetzbar betrachtet, wenn bestimmte Kriterien erfüllt sind, z.B. dass übereinstimmende Willenserklärungen vorliegen und dass ihre Abgabe weder unter Zwang noch durch Täuschung geschehen ist;

(iv) A und B erzielen Konsens über ein Geschäft, das die vom anwendbaren (d.h. dem von A und B gewählten) Recht geforderten Bedingungen für die Durchsetzung erfüllt.

Wenn das von A und B für anwendbar erklärte Recht als eine Bedingung für die Durchsetzbarkeit einer Transaktion einen Konsens zwischen den Parteien verlangt, so muss eine Einigung folglich auf zwei Ebenen vorliegen. Erstens müssen die Parteien sich über die Bedingungen der Vorvereinbarung geeinigt haben; und zweitens müssen sie sich über die Bedingungen des Hauptvertrages einig sein. Während die Dogmatik der Vorvereinbarungen alle notwendigen Bedingungen für eine wirksame »autorisierende « Einigung festlegt (indem sie bestimmt, wie frei, wie informiert usw. die Zustimmung sein muss), ${ }^{36}$ legen die Regeln des für anwendbar erklärten Rechts fest, wann eine Einigung im Hinblick auf eine rechtlich durchsetzbare Transaktion vorliegt. In diesem Sinne können wir von einer Vertragstheorie des doppelten Konsenses sprechen. Doch auch die Vertragsfreiheit ist in dieser Weise als zweischichtig zu denken: erstens, sofern die Parteien im Rahmen der Dogmatik der Vorabreden das auf ihre Geschäfte anwendbare Recht (oder Regeln oder Prinzipien) ${ }^{37}$ frei wählen können; und, zweitens, insofern sie Vertragsfreiheit innerhalb des gewählten Rechtsregimes genießen. Danach würde der Kern der Vertragsfreiheit gar nicht so sehr darin zu suchen sein, dass die Parteien ihre eigenen Vertrags- und Geschäftsbedingungen aufstellen dürfen, sondern vielmehr darin, dass ihnen die freie Wahl des anwendbaren Rechts gestattet ist.

Bevor wir zur Frage der Netzwerke zurückkehren, ist noch kurz auf den Schutz berechtigter Erwartungen der Vertragsparteien einzugehen. Wenn ein idealtypischer Fall

36 Vgl. Randy Barnett (1992) »The Sound of Silence: Default Rules and Contractual Consent«, 78 Virginia Law Review, 821-911.

37 Grundsätzlich sind die Vertragsparteien frei, Modellregeln wie die UNIDROIT Principles for International Commercial Contracts oder die Lando Principles zu wählen. Freilich muss jedes nationale Rechtssystem entscheiden, ob eine solche Rechtswahl vor den Zivilgerichten durchsetzbar sein soll. Durch die Verfügbarkeit solcher Modellregeln werden die Grenzen der Vertragsfreiheit ausgetestet (in Bezug auf die Vorvereinbarung). 
von prozeduraler Begründung vorliegt, weil A und B sich aus freien Stücken für »das englische Vertragsrecht « als anwendbares Recht entschieden haben, dann können sie vernünftiger Weise erwarten, dass die Regeln des englischen Vertragsrechts auf ihre Transaktion anwendbar sind. Auch das Konzept vernünftiger Erwartungen ist aber einer Zweischichtigkeit zugänglich. Denn es mag im englischen Vertragsrecht mehrere Regeln geben, die dem Schutz legitimer Erwartungen der Vertragsparteien zu dienen bestimmt sind und die in der einen oder anderen Weise dogmatisch fundiert sind - so ermittelt der neue Kontextualismus im Anschluss an Investors Compensation die vernünftigen Erwartungen der Parteien weniger anhand des Wortsinns des Vertrages als vielmehr anhand des intersubjektiven Verständnisses der Parteien. Entsprechendes gilt aber auch, sobald sich A und B aus freien Stücken auf ein bestimmtes Regime anwendbarer Regeln geeinigt haben; diese Regeln mögen dann dem Anliegen des Schutzes berechtigter Erwartungen Rechnung tragen oder nicht, und, wenn sie es tun, dann möglicherweise auf verschiedenen Wegen. Welchen Ansatz auch immer das anwendbare Recht selbst verfolgt, die grundlegendste Erwartung von A und B besteht darin, dass im Konfliktfall die von ihnen gewählten Regeln angewendet werden.

Das alles steht in bemerkenswertem Kontrast zu dem Fall, dass Rechtsregeln zum Schutz von berechtigten Erwartungen nach einem materiell-inhaltlichen Begründungsansatz gerechtfertigt werden (müssen). Im englischen Vertragsrecht lässt sich eine Entwicklungstendenz beobachten weg von einem statischen markt-individualistischen Ansatz (in dem die Vertragspraxis von Unternehmen sich an den aufgestellten Rechtsregeln orientieren soll) hin zu einem dynamischen markt-individualistischen Ansatz (in dem Rechtsregeln sich an der Vertragspraxis der Unternehmen orientieren und diese nachvollziehen). ${ }^{38}$ In dem erstgenannten Ansatz lenken die Regeln die Praxis (zumindest ist das die Intention); in dem letzteren lenkt die Praxis die Regeln. Unter dem erstgenannten erweist sich eine Erwartung als vernünftig, wenn sie auf eine Regel gestützt werden kann; unter der letzteren erweist sich eine Erwartung als vernünftig, wenn sie auf eine bestehende Praxis gestützt werden kann. Es besteht aber auch die Möglichkeit, dass die Dogmatik eine vernünftige Erwartung formuliert, die weder in geltenden Rechtsregeln noch in den Usancen bestimmter Wirtschaftsbranchen verankert werden kann, sondern nur in einem externen Standard, der die Ansprüche der Parteien bestimmt. ${ }^{39}$ Welcher Ansatz auch immer hinter einem konkreten Set von Regeln steht - wenn die Parteien sich nicht für die Anwendbarkeit jener Regeln entschieden haben, ist es Sache des Staates, eine materiell-inhaltliche Begründung dafür zu geben, dass er den Schutz von berechtigten Erwartungen verweigert oder in einer bestimmten Form gewährt.

Schließlich bleibt zu fragen, in welchem Verhältnis der Netzwerk-Gedanke zu diesen beiden Begründungsstrategien steht. Erstens, sofern A und B aus freien Stücken »das Recht der Netzwerke« gewählt haben, ist die Anwendung der Netzwerk-Regeln wie auch immer diese konkret aussehen mögen - prozedural gerechtfertigt (aber nicht

38 Vgl. Roger Brownsword (1996) »Static and Dynamic Market Individualism«, in: Roger Halson (Hg.) Exploring the Boundaries of Contract, Aldershot: Dartmouth, $48 \mathrm{ff}$.

39 Brownsword (Fn. 31) 103. 
automatisch auch materiell-inhaltlich). Zweitens, sofern das von A und B aus freien Stücken für anwendbar erklärte Recht - mag es das englische oder französische oder ein anderes sein - über eine Dogmatik der Netzwerke verfügt, dann ist die Anwendung der solchermaßen gewählten Regeln prozedural gerechtfertigt (aber nicht automatisch auch materiell-inhaltlich). Drittens, sofern sich A und B nicht auf irgend einen Set von anwendbaren Regeln geeinigt haben, steht der prozedurale Begründungsansatz nicht zur Verfügung. Viertens, mangels ihrer prozeduralen Begründbarkeit müssen die den Parteien auferlegten Regeln materiell gerechtfertigt werden - und zwar unabhängig davon, ob Netzwerk-Regeln direkt oder indirekt auferlegt werden (z.B. von einer an der Geschäftspraxis orientierten Dogmatik vernünftiger Erwartungen).

\section{Zwei Arten von Netzwerken}

In der Regel wird der prozedurale Begründungsansatz bei Verträgen zwischen Unternehmen herangezogen werden können, da sich diese Akteure bewusst - und oft unter professioneller Beratung - mit der Auswahl des für ihre Transaktionen anwendbaren Rechts befassen. Jedenfalls ist für den Bereich der Verbraucherverträge ein ähnlich breiter Einsatz der prozeduralen Begründungsstrategie schwer vorstellbar. Hier wird vielmehr der Staat den Parteien ein ganzes Paket von Rechten und Pflichten mit der Begründung vorgeben, es handele sich um einen inhaltlich angemessenen Ausgleich von Verbraucher- und Unternehmensinteressen. Sinnvoller Weise können wir also nur für unseren ersten Testfall (Unterverträge zwischen Bauunternehmern) die Möglichkeit einer prozeduralen Begründung des Netzwerks erörtern; in unserem zweiten Testfall (Reinigungsarbeiten für einen Verbraucher) müsste dagegen die Anwendung des Netzwerk-Gedankens materiell-inhaltlich gerechtfertigt werden. Wie sieht die Rechtfertigung jeweils konkret aus?

In beiden Fällen besteht das allgemeine Prinzip darin, die Verträge zwischen A und $B$ einerseits und B und C andererseits als Verträge eines Netzwerks von Verträgen anzusehen und diesen Umstand bei der Beurteilung von Fragen der Angemessenheit, der Risikoverteilung und ähnlichen im Streitfall auftretenden Fragen zu berücksichtigen. Negativ ausgedrückt, bedeutet das, dass der Bezugsrahmen für die Lösung von Streitigkeiten nicht auf die Beziehungen zwischen den Parteien eines bestimmten einzelnen Vertrages beschränkt ist (das wäre die bipolare Sichtweise). Positiv ausgedrückt, wird die Streitigkeit in dem Kontext des Netzwerks von Verträgen betrachtet, in dem sie entsteht (das Netzwerk stellt also den umfassenderen Bezugsrahmen für die Beurteilung des Streits dar). Abhängig von dem konkreten Rechtsregime, das auf die Geschäfte der Parteien anwendbar ist, lässt sich dieses allgemeine Netzwerk-Prinzip in einer Reihe von einzelnen Vorschriften konkretisieren - sei es, dass die Parteien selbst ein Netzwerk-Regime gewählt haben, sei es, dass das staatliche Recht eine Lösung über das Netzwerk vorgibt.

Im Bauprojekt-Testfall besteht das Problem darin, die Angemessenheit einer zwischen A und B ausgehandelten Klausel zu beurteilen, wenn es C (und nicht B) ist, der sich auf diese beruft. Wenn die Parteien eine Vorvereinbarung getroffen haben, nach der ein maßgeschneidertes Set von Netzwerk-Regeln auf ihre Geschäfte und mögliche 
Streitigkeiten Anwendung finden soll, so stellen diese Regeln nicht nur einen Bezugsrahmen dar, sondern anhand ihrer wird der Streit auch konkret entschieden. Da die Parteien der Anwendung dieser maßgeschneiderten Regeln auch zugestimmt haben, sind diese prozedural gerechtfertigt. Wenn die Parteien sich nicht auf ein Set von Netzwerk-Regeln als solches verständigt haben, sondern lediglich auf ein Rechtssystem, das den Netzwerk-Gedanken kennt und sich dieser in einzelnen Regelungen manifestiert, so ist die Anwendung des Netzwerk-Prinzips (wenn auch mit weniger vorhersehbaren Ergebnissen) ebenso prozedural gerechtfertigt. Denn in beiden Fällen bestehen zwischen A und C mit Rücksicht auf die Vorvereinbarung gleichsam »vorangegangene Geschäfte ; und es ist eben diese Vorvereinbarung, die die Parteien an die Netzwerk-Regeln bindet. Nur wenn die Parteien nicht bewusst ein bestimmtes Set von Regeln für anwendbar erklärt haben, müssen das Netzwerk-Prinzip oder konkretere Netzwerk-Vorschriften zwingend vorgegeben und entsprechend materiell-inhaltlich gerechtfertigt werden. Das leitet über zum Verbraucher-Testfall.

Der Verbraucher-Testfall berührt mehrere Probleme, die alle ihren Ursprung in den Geschäftsbeziehungen zwischen dem Verbraucher A und dem Reinigungsunternehmer B haben; vorliegend soll es jedoch gerade um einen Streit gehen, der zwischen A und dem Subunternehmer $\mathrm{C}$ entsteht. In einem solchen Fall muss die Anwendung des Netzwerk-Prinzips materiell-inhaltlich gerechtfertigt werden können. Sollte sich im Rahmen der rechtlichen Prüfung die Frage stellen, ob C sich auf eine Klausel aus dem Vertrag zwischen A und B berufen kann bzw. ob dies in Einklang mit den vernünftigen Erwartungen der Parteien steht, so würde es der Rückgriff auf das Netzwerk-Prinzip den Gerichten erlauben, die Frage im Kontext der miteinander zusammenhängenden Geschäftsbeziehungen aller Beteiligten zu behandeln. Auch wenn diese Methode nicht gerade die Vorhersehbarkeit von gerichtlichen Entscheidungen fördern wird, spricht doch entscheidend für sie, dass der Konflikt nunmehr in einem Kontext lokalisiert und behandelt werden kann, der weit eher der Wahrnehmung der Parteien selbst entspricht.

Was aber geschieht, wenn man die beiden Testfälle miteinander kombiniert? Stellen wir uns vor, dass die Bauprojektgesellschaft A dem Verbraucher Z ein fertiges Haus verkauft, dieses jedoch zuvor von Bauunternehmer B hat errichten lassen, der sich seinerseits des Subunternehmers $C$ bediente. Wie wir gesehen haben, würden einer prozedural gerechtfertigten Anwendung von Netzwerk-Regeln nur jene Beteiligten unterliegen, die sich durch eine entsprechende Vorvereinbarung gebunden haben. In unserem kombinierten Testfall würde dies bedeuten, dass A, B und C in einem Netzwerk miteinander verbunden sind, während $\mathrm{Z}$ kaum einer solchen Bindung unterliegen wird; im Gegenteil würde $\mathrm{Z}$ sich zu Recht beschweren, wenn auf ihn prozedural begründete Netzwerk-Regeln angewendet würden. In einer materiell-inhaltlich getragenen Anwendung von Netzwerk-Regeln (etwa eine solche, die die Interessen des $\mathrm{Z}$ als Verbraucher berücksichtigt) würde hingegen die Frage nicht lauten, ob Z, A, B oder C sich ein solches Regime selbst ausgesucht hätten, sondern ob seine obligatorische Anwendung mit guten Gründen gerechtfertigt werden kann.

Das führt auf eine weitere allgemeine Frage: Sofern in den Augen des Staates das Netzwerk-Prinzip der beste Weg ist, um einen angemessenen Interessenausgleich zwi- 
schen Hauskäufern wie Z und Projektentwicklern bzw. Bauunternehmern wie A, B und $\mathrm{C}$ herzustellen, wird die Freiheit der Letztgenannten zur Wahl eines Vertragsregimes, das keine Netzwerk-Regeln kennt, eingeschränkt werden müssen. Das entspräche den Fällen, in denen es den Parteien verboten wird, eine zwingende schadensersatzrechtliche Regelung abzubedingen. Hier eben zeigt sich, dass die erwähnte grundlegendere Dimension der Vertragsfreiheit in der Wahl eines Sets von anwendbaren Regeln liegt und weniger in der späteren Einigung über die eigentliche Transaktion.

\section{Fazit}

Als die Idee von Netzwerkverträgen zum ersten Mal ins Spiel gebracht wurde, gab es eine Reihe offener Fragen. Insbesondere war unklar, ob von einem Netzwerk nur dann auszugehen sei, wenn die Parteien dies beabsichtigt hatten, oder ob es auch dann anzunehmen sei, wenn mit seiner Hilfe (unabhängig vom Willen der Parteien) die tatsächliche Struktur der Transaktionen zwischen den Parteien am besten wiedergegeben werden konnte. Im Nachhinein wird deutlich, dass diese offenen Fragen den Unterschied zwischen einer von den Parteien frei gewählten Anwendung von Netzwerk-Regeln und der zwingenden Bindung an das Netzwerk-Prinzip betrafen. Deutlich wird auch, dass dieser Unterschied der weit wichtigeren Differenz zwischen prozedural rechtfertigungsfähigen und materiell-inhaltlich rechtfertigungsfähigen Verpflichtungen entspricht.

Verpflichtungen, die prozedural gerechtfertigt werden können, sind vertraglicher Natur. Wo Regelsets auf dem Konsens der Beteiligten beruhen, kann es sich nicht um Aberrationen vom Vertragsrecht handeln, sondern vielmehr um dessen eigentlichen Ausdruck. Wir befinden uns im Reich der Vertragsfreiheit (in Bezug auf die Festlegung der anwendbaren Regeln) und im Reich der Bindungswirkung von Verträgen (in Bezug auf das vereinbarte Rechtsregime).

In der Vergangenheit haben wir Verträge aus der falschen Perspektive, vom falschen Ende des Teleskops her betrachtet. Rechtswahlklauseln, Erklärung einer Geschäftsbesorgung nur aus Gefälligkeit, die Unterwerfung unter Netzwerk-Regime und ähnliches sind keine Ausnahmen, sondern geradezu Ausdruck vertragsrechtlichen Handelns, die das Wesen der vertraglichen Verpflichtung berühren. Im Gegensatz dazu herrscht auf den Massenmärkten für Verbraucher, wo es zur besinnungslosen Unterwerfung unter die lex loci kommt, der »Vertrag « oder das, was man so nennt, nur kraft Anordnung. An einen Vertrag mangels Wahrnehmung eines Opt-outs gebunden zu sein, kommt jedoch dem Tod des Vertrages gleich. Vielmehr ist die bewusste Entscheidung für den Vertrag der idealtypische Fall; und genau der tritt ein, wenn sich unternehmerische Akteure für ein Netzwerk-Regime entscheiden.

Allgemeiner ausgedrückt, ist ein auf Konsens gegründetes Netzwerk eine Miniaturversion der Idee des Gesellschaftsvertrages, oder besser: dessen Realität - nämlich ein Regime, das vom Konsens der Beteiligten autorisiert ist. Das Netzwerk ist weder merkwürdig noch ist es ein Ausnahmephänomen. Merkwürdig ist vielmehr, dass wir auch nach der Lockerung des Relativitätsprinzips fixiert bleiben auf den Vertrag als 
bipolare Transaktion und uns dabei Offensichtliches verborgen geblieben ist. Einerseits können sich die Vertragsparteien aus freien Stücken auf Netzwerkregeln einigen; andererseits kann das Netzwerk mitunter der angemessene Kontext für die Behandlung vertragsrechtlicher Streitigkeiten sein. 